\title{
Alfabetización digital en la educación básica en México: análisis documental del plan de estudios
}

\author{
Digital literacy in basic education in Mexico: documentary analysis of the curriculum \\ William René Reyes Cabrera
}

\begin{abstract}
RESUMEN
Esta investigación se inicia a partir del análisis de los documentos que integran el plan de estudios de educación básica en los Estados Unidos Mexicanos, con la finalidad de determinar el grado de integración de la alfabetización digital (AD). Para llevar a cabo esta investigación se definieron nueve dimensiones y se establecieron indicadores (palabras claves) que sirvieron como términos de búsqueda en los documentos oficiales. A partir de los registros analizados se observó que todas las dimensiones de la alfabetización digital consideradas se encontraban en el plan de estudios de nivel básico, siendo el pensamiento crítico la dimensión de mayor presencia y el pensamiento computacional la dimensión con menor presencia. Los resultados obtenidos permiten afirmar que la alfabetización digital se encuentra entre los puntos considerados importantes en la formación del estudiantado de nivel básico.

Palabras clave: alfabetización digital, educación básica en México, análisis documental, dimensiones.
\end{abstract}

\begin{abstract}
This research begins from the analysis of the archives that make up the curriculum of basic education in the United Mexican States, to determine the degree of integration of digital alphabetization (DA). To carry out this research, nine dimensions were defined, and indicators (keywords) established that served as search terms in official documents. From the analyzed records was observed that all the dimensions of digital alphabetization considered were found in the basic curriculum level, critical thinking being the dimension with the greatest presence and computational thinking the one with the least presence. The results obtained allow us to affirm that digital alphabetization is among the points considered important in basic education.

Keywords: digital literacy, basic education in Mexico, document analysis, dimensions.
\end{abstract}




\section{INTRODUCCIÓN}

La alfabetización digital (AD) está relacionada con el desarrollo de competencias en el uso de las tecnologías de la información y la comunicación (TIC); representa la habilidad de un individuo para realizar tareas de manera eficiente y eficaz en ambientes mediatizados por la tecnología. En este contexto se considera el uso de las computadoras, dispositivos móviles, la reproducción de datos e imágenes a través de programas computacionales aplicando y evaluando el nuevo conocimiento asociado a las TIC.

Los programas de AD deben estar orientados al desarrollo de habilidades asociadas al uso de las tecnologías, e integración de las TIC, como parte de las competencias básicas del estudiantado (UNESCO, 2017).

Valencia, Serna, Ochoa, Caicedo, Montes y Chavez (2016) afirman que

Con relación a las habilidades de aprendizaje transversales a cualquier dominio o área de conocimiento que el docente debe procurar desarrollar en los estudiantes, en concordancia con las principales perspectivas sobre Habilidades del Siglo XXI a nivel global se identifican las siguientes: pensamiento crítico, pensamiento creativo, comunicación y colaboración [p. 13].

Existen varios ejemplos en el mundo que han llevado a cabo programas de AD, tales como el proyecto Revitalización de la Alfabetización de Jóvenes y Adultos (RAYL en inglés) que se estableció durante el año 2015 en el estado de Cross River, en Nigeria, con la finalidad de lograr en los estudiantes competencias básicas de lectoescritura a través de medios digitales tales como computadoras y terminales móviles (UNESCO, 2017); el proyecto FATIH en Turquía, que tuvo como objetivo promover la igualdad de oportunidades en la educación y estimular la utilización de las TIC durante los procesos de aprendizaje (Yavuzalp, Derya, Curaoðlu, Durmus, Akayoðlu, Bahar, Kiliç y Tekinarslan, 2015), y en la República Oriental del Uruguay el proyecto socioeducativo creado en el año 2007 conocido como Plan Ceibal, que se propuso disminuir la brecha entre ciudadanos; dicho proyecto continúa en la actualidad, en estrecha colaboración con la Administración Nacional de Educación Pública, en su sede del Laboratorio Tecnológico del Uruguay (Rivoir, 2012). Por su parte, Corea del Sur cuenta desde el año 1985 con un sistema educativo basado en habilidades y ciudadanía digitales que se adaptan a las necesidades de la sociedad del conocimiento, lo que le ha permitido que en la actualidad sea uno de los países con mayor nivel de AD y adelantos tecnológicos en el mundo (Cho y Huh, 2017).

William René Reyes Cabrera. Profesor-investigador de la Facultad de Educación de la Universidad Autónoma de Yucatán, México. Es doctor en Educación y cuenta con especialidad en Entornos Virtuales de Aprendizaje por Virtual Educa (Argentina). Tiene reconocimiento al perfil PRODEP. Ha publicado en revistas de prestigio internacional en el área de educación y tecnologías como REIFOP (2019), Apertura (2020) y Eti@@net (2018 y 2021). Es miembro de la Red Interamericana de Liderazgo y Prácticas Educativas con sede en la Universidad Ciego de Ávila (Cuba). Correo electrónico: wreyes@correo.uady.mx. ID: https://orcid. org/0000-0002-3443-6385. 


\section{Programas de alfabetización digital en los Estados Unidos Mexicanos}

Desde finales del siglo XX se han implementado en los Estados Unidos Mexicanos diversos programas cuyo objetivo ha sido lograr el desarrollo de competencias digitales, cerrar brechas y democratizar el conocimiento; la mayoría de ellos se han desarrollado en la educación básica.

En el año 1985 se llevó a cabo el primer programa gubernamental de COOEENA (Computación Electrónica Básica) y posteriormente en los años ochentas y noventas se ejecutaron programas similares. Red Escolar en 1997, Enciclomedia en el 2004 y Habilidades Digitales para Todos en el 2009 (SEP, 2016) continuaron la serie, pero fue en el periodo 2012-2018 cuando se implementó una estrategia conocida como México Conectado (Gobierno de México, 2017), de la cual se desprendieron los programas MiCompu.mx y México Digital en el 2013,@aprende.mx en el 2014 y el proyecto de inclusión y alfabetización digital @prende 2.0 en el 2017 (SEP, 2016). Como se puede observar, en 23 años han existido al menos siete programas distintos relacionados con la AD, dificultando su continuidad, y en detrimento del desarrollo de capacidades digitales e informáticas de los estudiantes. Con respecto a los resultados e impactos obtenidos, la información encontrada estuvo relacionada con la cobertura y alcance de los programas, el acceso a estos recursos y el número de escuelas y estudiantes que se vieron beneficiados. Cárdenas (2016) se abocó a indagar, en los estados de Tabasco y Colima, sobre la infraestructura, capacitación de los docentes y la satisfacción de los estudiantes; por su parte García, Aquino y Ramírez (2016) y Tarango, Romo, Murguía y Ascencio (2014) analizaron el acceso que tienen los estudiantes a los recursos digitales en estados como Chihuahua, Tabasco y Veracruz. Kriscautzky y Ferreiro (2018), a través de estudios de caso en una escuela de la Ciudad de México, observaron el desempeño de los estudiantes en cuanto a sus habilidades de búsqueda de información en internet.

Si bien se han realizado trabajos de investigación como los nombrados anteriormente, queda por analizar cómo se está llevando a cabo, desde los planes de estudios oficiales, la vinculación con los programas nacionales de alfabetización digital para que incidan en los estudiantes y en sus competencias académicas (Cho y Huh, 2017; Anisimova, 2020).

El documento maestro del programa@prende 2.0 define áreas de oportunidad para investigar en el ámbito de la $\mathrm{AD}$ tales como el seguimiento de la formación en los niveles superiores, la evaluación de las habilidades digitales, las estrategias para el empleo de las TIC, la conectividad, el soporte técnico y la evaluación (SEP, 2016).

En esta investigación se consideró la necesidad de iniciar el proceso de estudio comenzando con el diagnóstico del estado actual de la alfabetización digital en el nivel básico del sistema educativo en los Estados Unidos Mexicanos, de forma tal que a través de la misma se puedan proponer intervenciones concretas y adecuadas 
para la formación inicial de estudiantes, favoreciendo el desarrollo de competencias digitales y el buen uso de las tecnologías en la educación. Es por ello que se comenzó a trabajar con una revisión sistemática sobre el tema para sentar las bases teóricas y se realizó una hoja de ruta para futuros estudios.

\section{MÉTOdOS Y MATERIALES}

La metodología de este estudio está enmarcada dentro del tipo cualitativo descriptivo. $\mathrm{Al}$ pensar en el diseño se estudiaron aquellos que combinaban estrategias de investigación cualitativas y cuantitativas. El análisis de los documentos, que corresponden a los planes oficiales del nivel básico en la educación de los Estados Unidos Mexicanos, se realizó teniendo en cuenta el enfoque descriptivo comparativo. La lectura de los trabajos de Barbosa, Barbosa y Rodriguez (2013); Instefjord y Munthe (2016); Norris, Van der Mars, Kulinna, Kwon y Amrein-Beardsley (2017), y Luna, Vicent, Reyes y Quiñonez (2019) fue la que determinó que el modelo sería apropiado teniendo en cuenta la naturaleza de la investigación.

\section{Objetivos de la investigación}

El objetivo general de esta investigación es determinar el grado de integración que tiene la alfabetización digital en los planes y programas de la educación básica en los Estados Unidos Mexicanos.

Se consideran los siguientes objetivos específicos:

- Identificar, a través de sus dimensiones, la presencia de la alfabetización digital en los planes y programas de la educación básica en los Estados Unidos Mexicanos.

- Determinar cuáles son los componentes de la educación básica, en los Estados Unidos Mexicanos, en los que tiene presencia la alfabetización digital.

\section{Contexto del campo: la educación básica}

En la Ley General de Educación (DOF, 2019) se establece que el sistema educativo mexicano lo componen los niveles de educación básica, media superior y superior, cada uno con sus características y modalidades. La educación básica, también conocida como educación inicial, se cursa en doce grados distribuidos en tres etapas: los tres primeros grados corresponden a la educación preescolar, los seis grados siguientes a la educación primaria y los tres últimos a la educación secundaria (SEP, 2017). En la figura 1 se representa de manera gráfica la distribución de las etapas y grados escolares de la educación básica en los Estados Unidos Mexicanos. 


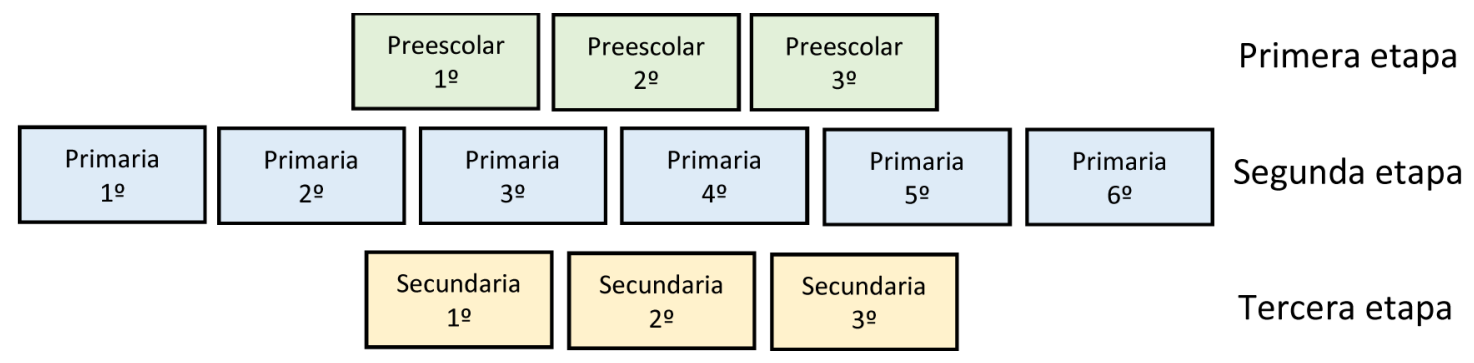

Figura 1. Etapas y grados de la educación básica en los Estados Unidos Mexicanos.

Fuente: Elaboración propia con información de SEP (2017).

Con respecto al currículo de educación básica, está basado en los llamados Aprendizajes clave para la educación integral (SEP, 2019) y se organiza en tres componentes curriculares, a saber: los campos de formación académica, las áreas de desarrollo personaly social, y los ámbitos de autonomía curricular. En la tabla 1 puede apreciarse de qué manera están organizados los componentes curriculares y los elementos que los constituyen.

Tabla 1. Organización curricular de educación básica en México.

Componentes curriculares

Campos de formación académica

Areas de desarrollo personal y social

mbitos de autonomía curricular
Elementos que lo constituyen

Lenguaje y comunicación

Pensamiento matemático

Exploración y comprensión del mundo natural y social

Artes
Educación socioemocional
Educación física
Ampliar la formación académica
Potenciar el desarrollo personal y social
Nuevos contenidos relevantes
Conocimientos regionales
Proyectos de impacto social

Fuente: Elaboración propia a partir de la información de SEP (2017).

Los campos de formación académica y las áreas de desarrollo personal y social son comunes en todo el país, en cambio los ámbitos de autonomía curricular se trabajan con base en proyectos concretos según la necesidad de cada estado y de cada zona.

\section{Los sujetos de la investigación: documentos de análisis}

La investigación se llevó a cabo mediante el análisis de los documentos correspondientes a los planes y programas oficiales vigentes en la educación básica en los Estados Unidos Mexicanos, que corresponden a las tres etapas y grados escolares.

A partir de la serie de documentos denominados Aprendiajes clave para la formación integral (SEP, 2017), en la cual se describe cada uno de los componentes curriculares, 
se seleccionaron veinte documentos para realizar su análisis, a saber: un documento correspondiente al plan de estudios, catorce documentos correspondientes a los campos de formación académica, cuatro documentos correspondientes a las áreas de desarrollo personal y social y un documento correspondiente a los ámbitos de la autonomía curricular. La tabla 2 permite observar la relación de los documentos considerados para su estudio.

Tabla 2. Relación de los documentos utilizados para la investigación.

Componente curricular

1. Plan de estudios
2. Campos de formación académica
2.1. Lenguaje y comunicación
2.2. Pensamiento matemático
2.3. Exploración y comprensión del
mundo natural y social

Documentos seleccionados

Aprendizaje clave

\author{
Introducción al campo 1: Lenguaje y comunicación \\ Programa: lengua extranjera, inglés \\ Programa: lengua materna, español \\ Programa: lengua materna indígena, segunda lengua indígena \\ Programa: segunda lengua, español
}

Programa: Matemáticas

Introducción al campo 3: Exploración y comprensión del mundo natural y social

Programa: Ciencia naturales y tecnología

Programa: Conocimiento del medio

Programa: Formación cívica y ética

Programa: Geografía

Programa: Historia

Programa: Historias, paisajes y convivencia en mi localidad

\begin{tabular}{ll}
\hline 3. Áreas de desarrollo personal y social & Introducción al área: Desarrollo personal y social \\
3.1. Artes & Programa: Artes \\
3.2. Educación socioemocional & Programa: Educación socioemocional \\
3.3. Educación física & Programa: Educación física \\
\hline 4. Ámbitos de autonomía curricular & Programa: Ámbitos de autonomía curricular \\
\hline
\end{tabular}

Fuente: Elaboración propia a partir de la información de SEP (2017).

\section{Instrumentos y procedimientos para la recolección de los datos}

La investigación se desarrolló en tres etapas. Durante la primera etapa se definieron las dimensiones de alfabetización digital, que se trabajarían teniendo en cuenta el programa@prende 2.0,y los referentes del mismo, denominados Marco Común Europeo de Competencia Digital Docente (INTEF, 2017) y Marco de Competencia Digital para Ciudadanos (Digicomp 2.0, 2016). Al mismo tiempo se tuvieron en cuenta otros documentos internacionales sobre el desarrollo de competencias digitales para el siglo XXI (Lemke, 2002; ISTE, 2016, y P21, 2017). De este análisis documental 
se definieron las nueve dimensiones que involucran la alfabetización digital en los Estados Unidos Mexicanos, a saber: pensamiento crítico, pensamiento creativo, manejo de la información, comunicación, colaboración, uso de la tecnología, ciudadanía digital, automonitoreo y pensamiento computacional. Para cada una de estas dimensiones se plantearon: el concepto, en forma textual como aparece en el programa de @prende 2.0 (SEP, 2016) y los descriptores o palabras clave (Miller y Salinas, 2019), que se utilizaron como términos de búsqueda (Luna, Vicent, Reyes y Quiñonez, 2019). Los descriptores se obtuvieron mediante el análisis de cada concepto. En la tabla 3 se presenta el desagregado de las dimensiones que componen la alfabetización digital en los Estados Unidos Mexicanos.

Teniendo en cuenta las dimensiones y sus descriptores se elaboraron fichas que tienen como finalidad recoger datos (Luna, Vicent, Reyes y Quiñonez, 2019; Quansah, Amoako y Ankomah, 2019), estableciendo un proceso sistemático en el registro de la información.

La segunda etapa de la investigación consistió en analizar cada uno de los documentos seleccionados (ver tabla 2) con la finalidad de determinar los descriptores de las dimensiones de la alfabetización digital; para lograr una mayor eficiencia y confiabilidad se convirtieron los documentos al formato electrónico PDF con la opción de reconocimiento óptico de caracteres (Optical Character Recognition u OCR) habilitada; posteriormente, y utilizando el programa Agent Ransack (Mythicsoft, 2019), se identificó cada descriptor a través de búsquedas booleanas.

La búsqueda arrojó un número de coincidencias entre todos los documentos, estos resultados se registraron de forma manual en la ficha de recogida de datos. Con algunas palabras clave fue necesario utilizar variantes debido a que con la palabra clave original no se obtuvieron suficientes datos. A estas variantes se las identificó a través del operador booleano OR; a fin de dar algún ejemplo es posible nombrar el descriptor respeto, que se trabajó con la expresión "respeta" OR "respetar". A fin de depurar el registro y dejar los descriptores que realmente inciden en las dimensiones de la AD, se realizó una segunda búsqueda utilizando el programa Agent Ransack. Se aplicaron criterios de inclusión y de exclusión y se realizó un análisis cualitativo de cada documento, tal como se observa en la tabla 4. Al finalizar el análisis se registraron los datos de forma manual en la ficha de recogida. La depuración del registro permitió agrupar los descriptores de dos formas, a saber: dimensiones de la alfabetización digital y componentes curriculares del plan de estudios de educación básica.

A manera de ejercicio se trabajó con la búsqueda, registro y depuración utilizando el nombre de cada una de las nueve dimensiones y se comparó con los resultados obtenidos.

La tercera etapa de la investigación consistió en el análisis de las dimensiones de la $\mathrm{AD}$ y de los componentes curriculares depurados que se encontraban en la ficha de recogida de datos. 
Tabla 3. Desagregado de dimensiones que componen la alfabetización digital en México.

\begin{tabular}{|c|c|c|c|}
\hline Dimensión & Definición & Descriptores & Fuentes consultadas \\
\hline Pensamiento crítico & $\begin{array}{l}\text { Proceso cognitivo que implica analizar, } \\
\text { comparar, inferir, sintetizar, interpretar } \\
\text { y evaluar los conocimientos adquiridos }\end{array}$ & $\begin{array}{l}\text { Analizar } \\
\text { Comparar } \\
\text { Sintetizar } \\
\text { Interpretar } \\
\text { Investigar }\end{array}$ & $\begin{array}{l}\text { SEP (2016, 2017), } \\
\text { P21Skills (2017), } \\
\text { INTEF (2017), } \\
\text { Castillo (2020) }\end{array}$ \\
\hline Pensamiento creativo & $\begin{array}{l}\text { La capacidad de aplicar el conocimiento obtenido } \\
\text { a fin de crear pensamientos, ideas o soluciones } \\
\text { nuevas y originales ante problemas reales }\end{array}$ & $\begin{array}{l}\text { Aplicar } \\
\text { Proponer } \\
\text { Desarrollar } \\
\text { Mejorar }\end{array}$ & $\begin{array}{l}\text { SEP (2016 y 2017), } \\
\text { Lemke (2002), } \\
\text { ISTE (2016), } \\
\text { P21Skills (2017), } \\
\text { INTEF (2017) }\end{array}$ \\
\hline Manejo de la información & $\begin{array}{l}\text { La capacidad de buscar la información, } \\
\text { evaluarla y aplicarla para resolver problemas }\end{array}$ & $\begin{array}{l}\text { Buscar } \\
\text { Evaluar } \\
\text { Seleccionar } \\
\text { Organizar }\end{array}$ & $\begin{array}{l}\text { Digicomp } 2.0 \text { (2016), } \\
\text { SEP }(2016,2017)\end{array}$ \\
\hline Comunicación & $\begin{array}{l}\text { Habilidad para utilizar medios y entornos digitales } \\
\text { que faciliten la comunicación y el trabajo colaborativo, } \\
\text { incluso a distancia; que promuevan el aprendizaje } \\
\text { individual y contribuyan al aprendizaje de otros }\end{array}$ & $\begin{array}{l}\text { Transmitir } \\
\text { Generar } \\
\text { Colaborar }\end{array}$ & $\begin{array}{l}\text { Lemke (2002), } \\
\text { ISTE (2016), } \\
\text { SEP (2016, 2017), } \\
\text { P21Skills (2017) }\end{array}$ \\
\hline Colaboración & $\begin{array}{l}\text { La capacidad para trabajar en grupo } \\
\text { a fin de conseguir un objetivo común }\end{array}$ & $\begin{array}{l}\text { Trabajar } \\
\text { Compartir } \\
\text { Decidir }\end{array}$ & $\begin{array}{l}\text { Digicomp } 2.0 \text { (2016), } \\
\text { SEP (2016, 2017), } \\
\text { P21Skills (2017) }\end{array}$ \\
\hline Uso de la tecnología & $\begin{array}{l}\text { La capacidad de usar herramientas tecnológicas } \\
\text { tales como hardware, software, Internet } \\
\text { y elementos periféricos para comunicarse, } \\
\text { colaborar, solucionar problemas y realizar tareas }\end{array}$ & $\begin{array}{l}\text { Cuidar } \\
\text { Seguridad } \\
\text { Identificar } \\
\text { Resolver } \\
\text { Dominio } \\
\text { Comunicar }\end{array}$ & $\begin{array}{l}\text { Digicomp } 2.0 \text { (2016), } \\
\text { SEP (2016, 2017), } \\
\text { P21Skills (2017) }\end{array}$ \\
\hline Ciudadanía digital & $\begin{array}{l}\text { La capacidad de comprender los asuntos humanos, } \\
\text { sociales y culturales en torno al uso y } \\
\text { aprovechamiento de las TIC, a fin de promover } \\
\text { conductas legales y éticas para comunicarse y } \\
\text { compartir a través de ambientes digitales }\end{array}$ & $\begin{array}{l}\text { Ética } \\
\text { Respeto } \\
\text { Protección } \\
\text { Distinguir } \\
\text { Comprender }\end{array}$ & $\begin{array}{l}\text { ISTE (2016), } \\
\text { SEP }(2016,2017) \text {, } \\
\text { P21Skills (2017), } \\
\text { Lauricella, Herdzina } \\
\text { y Robb (2020) }\end{array}$ \\
\hline Automonitoreo & $\begin{array}{l}\text { La aptitud de establecer metas de aprendizaje, } \\
\text { así como la planeación de estrategias para alcanzarlas }\end{array}$ & $\begin{array}{l}\text { Desempeño } \\
\text { Responsabilidad } \\
\text { Iniciativa }\end{array}$ & $\begin{array}{l}\text { Lemke }(2002) \text {, } \\
\text { SEP }(2016,2017)\end{array}$ \\
\hline Pensamiento computacional & $\begin{array}{l}\text { Es el proceso que trasciende el consumo } \\
\text { de TIC y deriva en la creación de herramientas } \\
\text { tecnológicas mediante un pensamiento } \\
\text { lógico, matemático y algorítmico }\end{array}$ & $\begin{array}{l}\text { Solucionar } \\
\text { Organizar datos } \\
\text { Representar datos } \\
\text { Transferir }\end{array}$ & $\begin{array}{c}\text { ISTE (2016), SEP } \\
(2016,2017), \text { P21Skills } \\
\text { (2017), Adell, Llopis } \\
\text { y Esteve (2019) }\end{array}$ \\
\hline
\end{tabular}


Tabla 4. Criterios de inclusión y exclusión utilizados para la depuración de los descriptores utilizados en la investigación.

Criterios de inclusión
El descriptor:
- Se orienta al desarrollo de una de las dimensiones de la AD
- Se alinea con la definición de una de las dimensiones de la
AD
- Se orienta a una acción, meta u objetivo que el estudiante
realizará durante su proceso de formación
- Se demuestra por parte del estudiante en términos
conductuales o de competencia

Fuente: Elaboración propia.

\section{Limitaciones y delimitaciones de la investigación}

La investigación en curso es la primera etapa de un proyecto sobre la alfabetización digital en estudiantes de nivel básico. En esta ocasión se analizó la información documental de los planes de estudios oficiales vigentes que incluyen preescolar, primaria y secundaria. Los libros de texto y los materiales didácticos que se utilizan se analizarán en investigaciones futuras.

\section{REsultados}

Se presentan los resultados del análisis realizado a los documentos del plan de estudios de educación básica en los Estados Unidos Mexicanos a partir de las nueve dimensiones de la AD.

La tabla 5 permite visualizar las frecuencias para cada dimensión de la alfabetización digital en los componentes curriculares del plan de estudios.

Al realizar el análisis de los datos presentados en la tabla 5 es posible observar que el pensamiento crítico es la dimensión con mayor presencia en los componentes curriculares con 616 registros, le siguen en importancia la ciudadanía digital con 296 registros y la dimensión colaboración con 212 registros. Automonitoreo y pensamiento computacional son las dimensiones con menor presencia, con 59 y 22 registros respectivamente.

En cuanto al componente campos de formación académica, es el que cuenta con mayor presencia en las dimensiones de $\mathrm{AD}$ con 1,152 registros, siguiendo en orden de importancia áreas de desarrollo personal y social con 562 registros, plan de estudios con 142 registros y ámbitos de la autonomía curricular con 12 registros. Los resultados del análisis de las tres dimensiones de los campos de formación académica se encuentran en la tabla 6 .

Al observar los datos que se encuentran en la tabla 6 , es posible afirmar que la dimensión pensamiento crítico es la de mayor presencia con 487 registros y en forma decreciente ciudadanía digital con 171 registros y manejo de la información con 124 
Tabla 5. Frecuencias para cada dimensión de la alfabetización digital en los componentes curriculares del plan de estudios de educación básica en México.

\begin{tabular}{|c|c|c|c|c|c|}
\hline \multirow[b]{2}{*}{ Dimensiones } & \multicolumn{4}{|c|}{ Componente curricular } & \multirow[b]{2}{*}{ Totales } \\
\hline & $\begin{array}{l}\text { Plan de } \\
\text { estudios }\end{array}$ & $\begin{array}{c}\text { Campos de } \\
\text { formación académica }\end{array}$ & $\begin{array}{c}\text { Áreas de desarrollo } \\
\text { personal y social }\end{array}$ & $\begin{array}{c}\text { Ámbitos de la } \\
\text { autonomía curricular }\end{array}$ & \\
\hline Pensamiento crítico & 25 & 487 & 98 & 6 & 616 \\
\hline Pensamiento creativo & 23 & 103 & 76 & 1 & 203 \\
\hline Manejo de la información & 9 & 124 & 43 & 0 & 176 \\
\hline Comunicación & 17 & 21 & 81 & 1 & 120 \\
\hline Colaboración & 9 & 102 & 99 & 2 & 212 \\
\hline Uso de la tecnología & 25 & 114 & 25 & 0 & 164 \\
\hline Ciudadanía digital & 21 & 171 & 102 & 2 & 296 \\
\hline Automonitoreo & 9 & 18 & 32 & 0 & 59 \\
\hline Pensamiento computacional & 4 & 12 & 6 & 0 & 22 \\
\hline Totales & 142 & 1,152 & 562 & 12 & 1,868 \\
\hline
\end{tabular}

Fuente: Elaboración propia.

Tabla 6. Frecuencias para cada dimensión de la alfabetización digital en los campos de formación académica del plan de estudios de educación básica en México.

\begin{tabular}{|c|c|c|c|c|}
\hline \multirow[b]{2}{*}{ Dimensiones } & \multicolumn{3}{|c|}{ Campos de formación académica } & \multirow[b]{2}{*}{ Totales } \\
\hline & $\begin{array}{c}\text { Lenguaje } \\
\text { y comunicación }\end{array}$ & $\begin{array}{c}\text { Pensamiento } \\
\text { matemático }\end{array}$ & $\begin{array}{c}\text { Exploración y comprensión } \\
\text { del mundo natural y social }\end{array}$ & \\
\hline Pensamiento crítico & 246 & 102 & 139 & 487 \\
\hline Pensamiento creativo & 37 & 12 & 54 & 103 \\
\hline Manejo de la información & 115 & 5 & 4 & 124 \\
\hline Comunicación & 11 & 2 & 8 & 21 \\
\hline Colaboración & 77 & 6 & 19 & 102 \\
\hline Uso de la tecnología & 39 & 62 & 13 & 114 \\
\hline Ciudadanía digital & 77 & 4 & 90 & 171 \\
\hline Automonitoreo & 9 & 1 & 8 & 18 \\
\hline Pensamiento computacional & 1 & 4 & 7 & 12 \\
\hline Totales & 612 & 198 & 342 & 1,152 \\
\hline
\end{tabular}

Fuente: Elaboración propia.

registros. Las dimensiones de menor presencia son pensamiento computacional, automonitoreo y comunicación, con 12, 18 y 21 registros respectivamente.

Con respecto a los campos de formación académica, es lenguaje y comunicación el que tiene mayor presencia, con 612 registros. Es de destacar que dicho campo lo integran las asignaturas Español, Lengua indígena e Inglés. Con menor cantidad de registros se encuentran Exploración y comprensión del mundo natural y social 
(integrado por las asignaturas Ciencias naturales y tecnología, Conocimiento del medio, Formación cívica y ética, Geografía e Historia), que muestra 342 registros, y pensamiento matemático (Matemáticas) con 198 registros.

Por otra parte, en el componente áreas de desarrollo personal y social se encontraron los resultados que se presentan en la tabla 7.

Tabla 7. Frecuencias para cada dimensión de la alfabetización digital en las áreas de desarrollo personal y social del plan de estudios de educación básica en México.

\begin{tabular}{|c|c|c|c|c|}
\hline \multirow[b]{2}{*}{ Dimensiones } & \multicolumn{3}{|c|}{ Áreas de desarrollo personal y social } & \multirow[b]{2}{*}{ Totales } \\
\hline & Artes & Educación socioemocional & Educación física & \\
\hline Pensamiento crítico & 48 & 37 & 13 & 98 \\
\hline Pensamiento creativo & 26 & 41 & 9 & 76 \\
\hline Manejo de la información & 33 & 4 & 6 & 43 \\
\hline Comunicación & 5 & 72 & 4 & 81 \\
\hline Colaboración & 25 & 72 & 2 & 99 \\
\hline Uso de la tecnología & 8 & 13 & 4 & 25 \\
\hline Ciudadanía digital & 25 & 65 & 12 & 102 \\
\hline Automonitoreo & 3 & 17 & 12 & 32 \\
\hline Pensamiento computacional & 2 & 2 & 2 & 6 \\
\hline Totales & 175 & 323 & 64 & 562 \\
\hline
\end{tabular}

Fuente: Elaboración propia.

El análisis de la tabla 7 permite inferir que en las áreas de desarrollo personal y social la dimensión de mayor presencia es ciudadanía digital con 102 registros, a la que continúan colaboración y pensamiento crítico con 99 y 98 registros respectivamente, siendo las de menor presencia el pensamiento computacional, el uso de la tecnología y el automonitoreo, con 6, 25 y 32 registros.

Con respecto a las áreas de desarrollo personal y social, el mayor número de registros lo presenta educación socioemocional con 323 registros, a la que le siguen artes con 175 registros y educación física con 64 registros. Cabe mencionar que estas áreas se abordan como asignaturas en todos los grados de educación básica.

Para finalizar se vuelcan los datos de las frecuencias para cada dimensión de la alfabetización digital en los componentes curriculares del plan de estudios de la educación básica en los Estados Unidos Mexicanos. En la tabla 8 se observan estos registros.

Luego de realizar el registro y depuración, teniendo en cuenta el nombre de cada una de las nueve dimensiones en los componentes curriculares, se pudo diferir que la dimensión con mayor presencia es la de comunicación con 65 registros, continuando en forma decreciente pensamiento crítico y colaboración, con 22 y 65 registros respectivamente. Las dimensiones con menor presencia fueron pensamiento creativo, 
Tabla 8. Frecuencias para cada dimensión de la alfabetización digital en los componentes curriculares del plan de estudios de educación básica en México.

\begin{tabular}{|c|c|c|c|c|c|}
\hline \multirow[b]{2}{*}{ Dimensiones } & \multicolumn{4}{|c|}{ Componente curricular } & \multirow[b]{2}{*}{ Totale } \\
\hline & $\begin{array}{l}\text { Plan de } \\
\text { estudios }\end{array}$ & $\begin{array}{c}\text { Campos de } \\
\text { formación académica }\end{array}$ & $\begin{array}{c}\text { Áreas de desarrollo } \\
\text { personal y social }\end{array}$ & $\begin{array}{l}\text { Ámbitos de la } \\
\text { autonomía curricular }\end{array}$ & \\
\hline Pensamiento crítico & 10 & 9 & 3 & 0 & 22 \\
\hline Pensamiento creativo & 1 & 1 & 0 & 0 & 2 \\
\hline Manejo de la información & 1 & 1 & 0 & 0 & \\
\hline Comunicación & 25 & 28 & 11 & 1 & 65 \\
\hline Colaboración & 3 & 2 & 12 & 0 & 17 \\
\hline Uso de la tecnología & 2 & 1 & 0 & 0 & 3 \\
\hline Ciudadanía digital & 1 & 1 & 0 & 0 & 2 \\
\hline Automonitoreo & 1 & 1 & 0 & 0 & 2 \\
\hline Pensamiento computacional & 2 & 2 & 0 & 0 & 4 \\
\hline Totales & 46 & 46 & 26 & 1 & 119 \\
\hline
\end{tabular}

Fuente: Elaboración propia.

manejo de la información, ciudadanía digital y automonitoreo, con 2 registros cada una de ellas. Los componentes curriculares plan de estudios y campos de formación académica fueron los que tuvieron mayor presencia en las dimensiones de alfabetización digital, con 46 registros correspondientemente.

\section{Discusión}

La alfabetización y las habilidades digitales son áreas que deben ser prioritarias de cara al presente y futuro de las generaciones de estudiantes de nivel básico quienes necesitan contar con competencias básicas, y blandas, para competir en un mundo cada vez más globalizado y tecnificado (Digicomp 2.0, 2016; ISTE, 2016). La identificación en los planes oficiales de educación de la alfabetización digital mediante sus dimensiones permite conocer cómo las mismas se integran al currículo tanto en las asignaturas como en las demás áreas de formación (Adell, Llopis, Esteve y Valdeoliva, 2019). Es necesario tener en cuenta que el plan de estudios de nivel básico atiende la formación que resultará vital para continuar con solvencia los siguientes niveles educativos.

A partir del análisis documental se ha podido observar que, de los componentes curriculares analizados, la mayor presencia de las dimensiones de alfabetización digital se encuentra en los campos de formación académica; es de considerar que en este componente se incluye el mayor porcentaje de asignaturas que se cursarán durante los doce años de trayectoria educativa en el nivel básico, describiéndose en cada plan el enfoque pedagógico, las orientaciones didácticas, el contenido temático 
y las sugerencias de evaluación, entre otros puntos. Continúan en presencia las áreas de desarrollo personal, distribuidas en tres asignaturas con características similares en sus planes y programas. En cuanto a la menor presencia de la alfabetización digital, se observa en el plan de estudios y en los ámbitos de la autonomía curricular. Los documentos correspondientes a estos componentes son de carácter conceptual histórico, y de directrices pedagógicas y administrativas con un enfoque tanto en el estudiante como en el profesor, la familia y la escuela.

Por último, en relación con las dimensiones de alfabetización digital presentes en los planes y programas de nivel básico es posible observar que cuenta con mayor presencia el pensamiento crítico con 616 registros, al que siguen ciudadanía digital con 296 registros y colaboración con 212. El pensamiento crítico es una dimensión que, se puede decir, va más allá de la alfabetización digital, ya que es vital para el currículo (en especial de nivel básico); incluye el pensamiento de orden superior que facilita el juicio y se basa en criterios correctivos y sensibles al contexto (Castillo, 2020). Debido a estas características es adecuado que se desarrolle en los tres campos de formación académica y en especial en el de lenguaje y comunicación. La ciudadanía digital también es considerada como una dimensión que trasciende lo digital (Lauricella, Herdzina y Robb, 2020), debido a que en ella se abordan comportamientos éticos, de respeto y de tolerancia, entre otros, que son vitales para la sana convivencia. En los documentos de Aprendizajes clave se hace referencia explícita a los mismos. El pensamiento computacional tiene poca presencia en el plan de estudios de educación básica. Esteve-Mon, Llopis y Adell (2020) afirman que esta dimensión es fundamental en la alfabetización y competencia digital, ya que permite abordarla como una herramienta para la solución de problemas a través de la tecnología (Adell, Llopis, Esteve y Valdeolivas, 2019).

\section{CONClUSIONES Y TRABAJOS FUTUROS}

Los resultados encontrados evidencian una considerable integración entre los planes oficiales de educación con el programa nacional de AD (@prende 2.0), teniendo la mayor presencia en los campos de formación académica y específicamente en lenguaje y comunicación. Sin embargo, al valorar la presencia de cada una de las dimensiones de AD se observa que esta es dispar, mientras que pensamiento crítico tiene 616 registros, pensamiento computacional tiene 22 registros. Es evidente la diferencia entre la dimensión con mayor presencia, pensamiento crítico, con la dimensión pensamiento creativo, que le sigue en orden decreciente con 203 registros. Esta investigación es el punto de partida a través del cual se lleva a cabo el proyecto que tiene como objetivo identificar el nivel de alfabetización digital en los estudiantes del nivel secundario en el estado de Yucatán. El análisis de los documentos permitió reconocer, en los documentos oficiales de educación básica de los Estados Unidos Mexicanos, la presencia 
de las dimensiones de alfabetización digital; resta por analizar las habilidades digitales de los estudiantes y la forma en la cual los profesores las incentivan.

\section{Agradecimientos}

Al Consejo Nacional de Ciencia y Tecnología (CоnACyT) por el financiamiento del proyecto A1-S-9847 "La alfabetización digital en las y los estudiantes de nivel secundaria en el estado de Yucatán”, proyecto que se originó con base en la investigación que se trabajó en este artículo.

A la Facultad de Educación de la Universidad Autónoma de Yucatán por las facilidades técnicas y administrativas para la realización del proyecto.

A la Secretaría de Educación del Gobierno del Estado de Yucatán (SEGEY) por su interés y apoyo que permitieron llevar a cabo la investigación.

\section{REFERENCIAS}

Adell, J., Llopis, M., Esteve, F., y Valdeolivas, M. (2019). El debate sobre el pensamiento computacional en educación. RIED. Revista Iberoamericana de Educación a Distancia, 22(1), 171-186. https://doi.org/10.5944/ ried.22.1.22303.

Anisimova, E. (2020). Digital literacy of future preschool teachers. Journal of Social Studies Education Research, 11(1), 230-253. Recuperado de: https:// files.eric.ed.gov/fulltext/EJ1251924.pdf.

Barbosa, J., Barbosa, J., y Rodriguez, M. (2013). Revisión y análisis documental para estado del arte: una propuesta metodológica desde el contexto de la sistematización de experiencias educativas. Investigación bibliotecológica, 27(61), 83-105.

Cárdenas, M. (coord.) (2016). Evaluación del Programa Inclusión y Alfabetización Digital (PIAD). Recuperado de: http://bit.ly/2pzzK35.

Castillo, R. (2020). El pensamiento crítico como competencia básica. Una propuesta de nuevos estándares pedagógicos. IXTLI - Revista Latinoamericana de Filosofía de la Educación, 7(14), 127-148.

Cho, J., y Huh, J. (2017). UNESCO. New education policies and practices in South Korea. Recuperado de: http:// bit.ly/2pAes5y.

Digicomp 2.0 (2016). DigiComp 2.0: The digital competence framework for citizens. Update Phase 1: The conceptual reference model. https://doi.org/10.2791/11517.
DOF (2019). Ley General de Educación. Recuperado de: http://www.diputados.gob.mx/LeyesBiblio/pdf/ LGE_300919.pdf.

Esteve-Mon, F., Llopis, M., y Adell, J. (2020). Digital competence and computational thinking of student teachers. International Journal of Emerging Technologies in Learning, 15(2), 29-40. https://doi.org/10.3991/ijet. v15i02.11588.

García, V., Aquino, S., y Ramírez, N. (2016). Programa de alfabetización digital en México: 1:1. Análisis comparativo de las competencias digitales entre niños de primaria. CPU-e. Revista de Investigación Educativa, (23), 24-44. Recuperado de: http://bit.ly/2DOMVBH.

Gobierno de México (2017). Informe final del Programa "México Conectado". Recuperado de: https://bit. ly/2XtN2xT.

Instefjord, E., y Munthe, E. (2016). Preparing pre-service teachers to integrate technology: An analysis of the emphasis on digital competence in teacher education curricula. European Journal of Teacher Education, 39(1), 7793. https://doi.org/10.1080/02619768.2015.1100602.

INTEF [Instituto Nacional de Tecnologías Educativas y de Formación del Profesorado] (2017). Marco común de competenciadigital docente, octubre 2017 . Recuperado de: https://bit.ly/3E4qE2Y.

ISTE (2016). ISTE standards for students. Recuperado de: https://bit.ly/3nlsjeL. 
Kriscautzky, M., y Ferreiro, E. (2018). Evaluar la confiabilidad de la información en Internet: cómo enfrentan el reto los nuevos lectores de 9 a 12 años. Perfiles Educativos, 40(159), 16-34. Recuperado de: https:// bit.ly/3lgbu1Q.

Lauricella, A., Herdzina, J., y Robb, M. (2020). Early childhood educators' teaching of digital citizenship competencies. Computers \& Education, 158, 1-9. https:// doi.org/10.1016/j.compedu.2020.103989.

Lemke, C. (2002). en Gange 21 st century skills: Digital literacies for a digital age. Recuperado de: https://bit.ly/2X5JYfN.

Luna, U., Vicent, N., Reyes, W., y Quiñonez, S. (2019). Patrimonio, curriculum y formación del profesorado de educación primaria en México. Revista Electrónica Interuniversitaria de Formación del Profesorado, 22(1). https://doi.org/10.6018/reifop.22.1.358761.

Miller, A., y Salinas, C. (2019). A document analysis of student conduct in Florida's Community Colleges. Community College Journal of Research and Practice, 43(1011), 796-802. https://doi.org/10.1080/10668926.20 19.1600606.

Mythicsoft (2019). Agent Ransack. Recuperado de: https:/ / bit.ly/2X9JwfP.

Norris, J., Van der Mars, H., Kulinna, P., Kwon, J., y Amrein-Beardsley, A. (2017). A document analysis of teacher evaluation systems specific to Physical Education. The Physical Educator, 74(4). https://doi. org/10.18666/TPE-2017-V74-I4-7509.

P21 (2017). 21 st century skills. Recuperado de: https://bit. ly/2Xd3Qxs.

Quansah, F., Amoako, I., y Ankomah, F. (2019). Teachers' test construction skills in Senior High Schools in
Ghana: Document analysis. International Journal of Assessment Tools in Education, 6(1), 1-8. http:/ /dx.doi. org/10.21449/ijate.481164.

Rivoir, A. (2012). Cinco años del Plan Ceibal. Algo más que una computadora para cada niño. Recuperado de: https://bit.ly/2Qx6Q1N.

SEP (2016).Programa@aprende 2.0. México. Recuperado de: https://bit.ly/3txHtyE.

SEP (2017). Aprendizajes clave para la educación integral. Recuperado de: https://bit.ly/3hkxXtN.

SEP (2019). Acuerdo 20/11/19 por el que se establece los planes y programas de estudio de nivel básico. Recuperado de: https://bit.ly/3jZPaKz.

Tarango, J., Romo, J., Murguía, L., y Ascencio, G. (2014). Uso y acceso a las TIC en estudiantes de escuelas secundarias públicas en la ciudad de Chihuahua, México: inclusión en la didáctica y en la alfabetización digital. Alfabetización Digital, 25(1), 133-152. http:/ / dx.doi.org/10.5209/rev_RCED.2014.v25.n1.41250.

UNESCO (2017). El aprendiraje digital mejora los medios de subsistencia en Nigeria. Recuperado de: http://bit. ly/2DPIvub.

Valencia, T., Serna, A., Ochoa, S., Caicedo, A., Montes, J., y Chávez, J. (2016). Competencias y estándares TIC desde la dimensión pedagógica: una perspectiva desde los niveles de apropiación de las TIC en la práctica educativa docente. Pontificia Universidad Javeriana. Recuperado de: https://bit.ly/3nnp5Hw.

Yavuzalp, N., Derya, M., Curaoðlu, O., Durmus, S, Akayoðlu, S., Bahar, M., Kiliç, F., y Tekinarslan, E. (2015). FATIH Project in Turkey: A case analysis. International Journal of Research in E-learning, 1(1), 117-127.

Cómo citar este artículo:

Reyes Cabrera, W. R. (2021). Alfabetización digital en la educación básica en México: análisis documental del plan de estudios. IE Revista de Investigación Educativa de la REDIECH, 12, e1155. doi: 10.33010/ie_rie_rediech.v12i0.1155. 